\title{
Risk of homelessness was increased in schizophrenia when 3 factors were present
}

\author{
Olfson M, Mechanic D, Hansell S, et al. Prediction of homelessness within three months of discharge among inpatients with \\ schizophrenia. Psychiatr Serv 1999 May;50:667-73.
}

\section{Question}

Are there factors that place inpatients with schizophrenia at an increased risk of becoming homeless after discharge from the hospital?

\section{Design}

Inception cohort (defined by point of hospital discharge) followed up for 3 months.

\section{Setting \\ New York City, New York, USA.}

\section{Patients}

316 English speaking inpatients between 18 and 64 years of age with schizophrenia or schizoaffective disorder who were eligible for or enrolled in Medicaid and who were subsequently discharged from general hospitals. Exclusion criteria were a severe and highly disabling general medical condition, length of stay in hospital >120 days, discharge against medical advice, or transfer to another inpatient psychiatric facility. 263 patients $(83 \%)$ were followed up for 3 months.

\section{Assessment of prognostic factors}

Data on sociodemographic (including legal history) and clinical characteristics (clinical symptoms assessed using the Brief Psychiatric Rating Scale [BPRS], global functioning assessed using the Global Assessment Scale [GAS], and substance use disorders) were collected at the time of hospital discharge.

\section{Main outcome measure}

Episode of homelessness defined as living on the street or in a park, bus station, abandoned building, or similar setting.

\section{Main results}

20 patients $(7.6 \%)$ reported a period of homelessness during the 3 month follow up period. In a logistic regression analysis controlling for age, sex, and race, BPRS score at hospital discharge was associated with subsequent homelessness. A diagnosis of a substance use disorder was associated with an sixfold increase in the risk of becoming homeless (adjusted odds ratio [OR] 6.1, CI 2.0 to 18.4). A 1 point increase in BPRS score increased the relative risk (RR) of becoming homeless by $6.5 \%$ (adjusted OR 1.07, CI 1.02 to 1.11). For each 1 point decrease in GAS score at hospital discharge, the RR of becoming homeless increased by $5.9 \%$ (adjusted OR 1.06, CI 1.01 to 1.10).

\section{Conclusion}

In patients with schizophrenia, the risk of becoming homeless after hospital discharge was increased for those who had a comorbid drug use disorder, increased psychiatric symptoms, or poor global functioning.

Sources of funding: Robert Wood Johnson Foundation and the National Institute of Mental Health. For correspondence: Dr M Olfson, Department of Psychiatry, Columbia University College of Physicians and Surgeons, New York State Psychiatric Institute, 1051 Riverside Drive, New York, NY 10032, USA. Fax +12125683534.

\section{Commentary}

An increase in homelessness among patients with schizophrenia is one of the tragic downsides of deinstitutionalisation, a humanistic process that in many countries often proceeded faster than alternative facilities could be arranged.

The subsequent growing need for community based services has culminated in a few well researched trials showing the effectiveness of several case management interventions in improving social outcomes such as homelessness. ${ }^{1}$ These intensive interventions, some of which are recommended by the findings of the Schizophrenic Patients Outcomes Research Team findings, ${ }^{2}$ are not less expensive than institution based care and therefore should be targeted at high risk people. The study by Olfson $e t$ al it is important because it identifies high risk people and therefore appropriate therapeutic strategies could be tailored to mitigate homelessness.
The cohort design used in this study provides the best information we have about the risk of homelessness, but the authors acknowledge that other factors may mediate the loss of housing. Future research could build on this study to elicit other risk factors that contribute to this multidimensional problem.

There is no direct reference to the homogeneity of the illness stage of patients participating in this study. Heterogeneity of illness stage could obscure conclusions when testing hypotheses about predictions of course or outcome. This solid hypothesis driven study, however, is superior to previous work, and excels in evaluating its limitations inherent to studying complex issues in this highly recidivistic population.

Inevitably, the question arises whether atypical anti-psychotics could alleviate the incidence of homelessness. An independent study based on the design of this study could confirm or refute some revolution- ary claims made by the pharmaceutical industry.

The authors' comments that the problems faced by homeless people with schizophrenia cannot be solved by the mental health system alone is underscored by a pertinent editorial in the same journal: we can always use more research to refine models of housing and of service integration. But what we really need is leadership to put in place what we already know. ${ }^{3}$

\section{Wim Verbeeck, MB Bch, FcPsych Tara Hospital Johannesburg, South Africa}

1 Mueser KT, Bond GR, Drake RE, et al. Models of community care for severe mental illness: a review of research on case management. review of research on case
Schizophr Bull 1998;24:37-74.

2 Lehman AF, Steinwachs DM. Translating reLehman AF, Steinwachs DM. Translating research into practice: the schizophrenia patient outcomes research team (PORT) treatment recommendations. Schizophr Bull 1998;24:110.

3 Drake RE, Wallach MA. Homelessness and mental illness: a story of failure (editorial). Psychiatr Serv 1999;50:589. 